Progress in Flight Physics 5 (2013) 457-480

DOI: $10.1051 /$ eucass/201305457

(C) Owned by the authors, published by EDP Sciences, 2013

\title{
A NOVEL KINETIC MODEL FOR CHEMICAL ENERGY ACCOMMODATION ON SILICA SURFACE
}

\section{S. Di Benedetto ${ }^{1}$ and C. Bruno ${ }^{2}$}

\author{
${ }^{1}$ CIRA, Italian Aerospace Research Center \\ Via Maiorise, Capua 81043, Italy \\ ${ }^{2}$ UTRC
}

411 Silver Lane, MS 129-29, East Hartford, CT 06118-1127, USA

\begin{abstract}
A new phenomenological catalysis model for silica surface has been developed, the main feature of which is the capability of accounting for chemical energy accommodation (CEA), i. e., the effective transfer of chemical energy to the catalyst surface by catalytically formed molecules. In this new model the interaction time between a just-formed molecule and the surface is compared with the time of other processes, and the efficiency of the interaction depends on the coupling between the molecules interaction time before desorption and the phonon frequency distribution (a characteristic period of surface vibration). These two quantities have been evaluated for the case of oxygen atoms impacting a silica surface. To this aim, a new three dimensional model of the interaction potential between the surface atoms and the impacting atom has been built and utilized inside the bulk structure of crystalline silica. Finally, the model has been validated using data collected during a test campaign in the CIRA SCIROCCO Plasma Wind Tunnel (PWT) on a full-scale aerodynamic spacecraft component.
\end{abstract}

\section{INTRODUCTION}

Gas-surface interaction phenomena play a fundamental role in modeling and simulation of atmospheric reentry conditions. In particular, the energy released on the surface by catalyzed chemical reactions of oxygen and nitrogen atoms contributes to the vehicle surface heat load for a significant part of the total; therefore the study of catalytic recombination in atmospheric re-entry conditions is of fundamental interest for the design of thermal protection systems (TPS) for space vehicles. 
Among different numerical approaches to the study of catalysis, the kinetic, or phenomenological, is pursued in this work; it allows to go deeply into the physics of the problem, but, simultaneously, provides a more economical and faster mean than more fundamental approaches and thus can be used for engineering purposes.

An issue has been identified as priority in the existing catalysis modeling: that is the capability of accounting for CEA, i. e., the effective transfer of chemical energy to the catalyst surface by catalytically formed molecules. In fact, if justrecombined molecules leave the surface in an excited state, only a fraction of their recombination energy would be deposited on the surface. The parameter defining this fraction in the kinetic models is the chemical energy accommodation coefficient, $\beta$, defined as

$$
\beta=\frac{\text { heat flux released at surface }}{\text { heat flux of recombination }}=\frac{\dot{q}}{(\Delta \dot{n} / 2)} D
$$

where $\Delta \dot{n}$ is the number of atoms "consumed" by the unit surface in the unit time; therefore, $\Delta \dot{n} / 2$ is the flux of recombined molecules, and $D$ is their dissociation energy.

Once the chemical energy accommodation coefficient is known, the recombination coefficient $\gamma$ can be replaced by the effective recombination coefficient $\gamma^{\prime}=\gamma \beta$, a very important quantity, since it defines the catalytic heat flux to a wall, not only as the result of the recombination mechanisms, but also of the effective heat transferred to the surface during the recombination.

Many experimental findings and calculations have shown that CEA is not related in a simple way to temperature, recombination mechanism, density of adsorbed atoms, and possibly to other factors. Said otherwise, this is a case where the scientific community does not even know what are the unknowns.

For this reason, most of the heterogeneous catalysis models assume full accommodation $(\beta=1)$, thus providing a conservative estimate of the heat flux at the wall. (This strategy has resulted in wrong density and pressure coefficient predictions during the first Shuttle reentry.)

This said, the aim of this paper is to identify at least some of the parameters that mainly influence the transferring of energy to the surface, and then to derive a simplified expression of the chemical accommodation coefficient, so as to improve our capability to numerically predict the heat flux deposited on a TPS during reentry.

The first attempt to derive an analytical expression of the CEA coefficient was done in the past by Halpern and Rosner [1] with their definition of a 'minimum value,' equivalent to assume that just-formed molecules transfer their energy on a time scale long compared with that of the other processes. These authors, in fact, assumed that ER (Eley-Rideal mechanism) forms excited molecules desorbing very fast (a gas/surface collision lasts for about a picosecond) so that molecules 
have very little time to exchange their excess internal energy with the surface. Accordingly, they predicted that the E-R mechanism will result in a very low CEA.

The Langmuir-Hinshelwood (LH) mechanism, instead, should lead to an almost complete accommodation, i. e., $\beta \sim 1$.

The simple model by Halpern and Rosner was the starting point for our new modeling; first, it was modified in order to comply with the kinetic catalysis model developed in the past by Nasuti et al. for silica surfaces [2], then it was completed in order to account for the extra release of energy to the surface due to the possible coupling between the molecule desorbing frequency and that of the lattice phonons. This point will be the subject of the following sections.

\section{MODELING CHEMICAL ENERGY ACCOMMODATION}

\subsection{The Minimum $\beta$ Value}

For a first estimate of $\beta$, Halperin and Rosner [1] found its minimum value when recombined molecules desorb without depositing the energy on the surface. This is the case where only adsorption is responsible for the energy deposition, its adsorption energy transmitted to the solid on a time scale short compared to the other processes. This minimum value of $\beta$ was calculated in [1] and is reported hereinafter:

$$
\beta_{\min }=\frac{(1 / 2) Q_{m} \mu(1-\theta)^{2}+Q_{a}(1-\theta)-Q_{R} \gamma^{*} \theta-(1 / 2) Q_{\mathrm{LH}} \sigma \theta^{2}}{\gamma D / 2}
$$

where $\mu$ is the ratio between molecular and atomic flux $\left(Z_{m} / Z_{a}\right), \gamma^{*}$ is the microscopic Rideal recombination coefficient, and $\sigma$ is defined as

$$
\sigma=v n_{1} Z_{m}^{-1} \exp \left(-\frac{Q_{\mathrm{LH}}}{k T}\right)
$$

The first two terms in the numerator of Eq. (1) are the energies of molecules adsorption (dissociative adsorption) and atoms adsorption from which the energies needed for the activation of the two recombination processes (the last two terms in the numerator) must be subtracted.

This expression has been modified in order to comply with the kinetic catalysis model developed by Nasuti et al. [2] for silica surfaces. In particular:

- the dissociative adsorption (molecules adsorption) is assumed negligible with respect to the atomic one; 


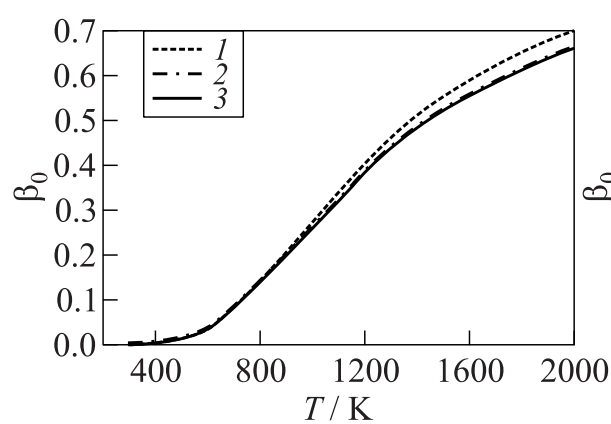

(a)

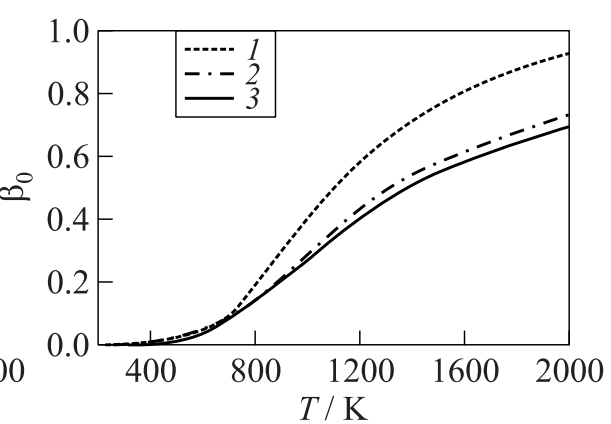

(b)

Figure 1 Dependence of $\beta_{\min }$ on temperature; effect of $\mathrm{O}$ partial pressure at fixed nitrogen partial pressure: $(a) p_{\mathrm{N}}=10 \mathrm{~Pa} ;(b) p_{\mathrm{N}}=100 \mathrm{~Pa} ; 1-p_{\mathrm{O}}=100 \mathrm{~Pa} ; 2-$ 500 ; and $3-p_{\mathrm{O}}=1000 \mathrm{~Pa}$

- the sticking coefficient $s_{a}$ is different from 1 , and has been introduced in the evaluation of the energy released by adsorbed atoms and in the total energy of recombination;

- desorption of atoms is considered a further mechanism which "takes" energy from the surface that must be added to the numerator of Eq. (1); and

- the presence of $\mathrm{OH}$ surface contamination is modeled, with the result that some surface sites (modeled by a coverage fraction $\theta_{\mathrm{OH}}$ ) are not free, that is, are not available for atoms adsorption.

Under these assumptions, the expression for $\beta$ has been reformulated as follows:

$$
\beta_{\min }=\frac{Q_{a} s_{a}\left(1-\theta-\theta_{\mathrm{OH}}\right)-Q_{R} \gamma^{*} \theta-(1 / 2) Q_{\mathrm{LH}} \sigma \theta^{2}-Q_{a} \delta \theta}{\left[s_{a}(1-\theta)-\delta \theta+\gamma^{*} \theta\right] D / 2} .
$$

This expression has been introduced in the kinetic model for a TPS based on an exposed silica surface, with the final recombination coefficient thus defined by $\gamma^{\prime}$.

In Fig. 1, $\beta_{\mathrm{O} \text { min }}$ (minimum chemical energy accommodation for $\mathrm{O}$ recombination) from Eq. (2) is plotted as a function of temperature, at various oxygen partial pressure; here the nitrogen partial pressure is kept constant. The parameter $\beta_{\mathrm{O} \text { min }}$ shows a monotonic increase with temperature; this is consistent with the idea that high accommodation levels are associated with $\mathrm{LH}$ recombination and that this latter is enhanced as temperature increases. Moreover, the distribution shows that $\beta_{\mathrm{O} \text { min }}$ increases with increasing nitrogen partial pressure $\left(p_{\mathrm{N}}\right)$ and decreases with increasing oxygen partial pressure $\left(p_{\mathrm{O}}\right)$. 


\subsection{Discussion of the Chemical Energy Accomodation Process}

The chemical accommodation coefficient introduced in Eq. (2) is just a minimum value; in some circumstances the molecule just recombined can transfer to the surface some "extra" energy. The mechanisms which influence and favor this extra release of energy are still unclear; understanding these mechanisms is crucial to the final aim of the present research activity, that is, the capability to predict their effect on the heat release and the temperature of the surface. To this purpose much effort has been done in order to identify the parameters that might have a role in the exchange of energy between gas and surface in catalytic processes.

While reading and comparing the literature about CEA and looking at the physics of this phenomenon, three issues seem to play an important role in accommodation:

1. The kinetic energy of the atoms impacting the surface.

Although this parameter has intuitively an important role in the definition of $\beta$, the effect(s) due to accommodation when increasing the kinetic energy of the impacting atom is/are not clear. An in-depth analysis of this dependence is that by Cacciatore et al. [3]. Results of molecular dynamics show that increasing kinetic energy (increasing macroscopic temperature) generally leads to a higher transfer of energy to the surface, but, at the same time, the collisional regime reduces the probability an atom has to react: the tendency for an atom is to be trapped or to be scattered back to the gas phase. Thus there should be a point when chemical energy accommodation inverts its dependence on kinetic energy. Note that in the expression for the chemical energy accommodation formulated in [4] the velocity of the atom impacting the surface appears at the denominator.

2. The lifetime of the molecule before desorption. This point is commonly accepted: the increase of $\beta$ in a recombination mechanism depends on the lifetime of the molecule formed before desorption. Halpern and Rosner [1] suggest that it is the time associated with the two recombination processes (ER and LH) that ensures the higher release of energy to the surface in the case of LH recombination (the slower of the two mechanisms).

3. The coupling between the vibrational frequency of recombining molecule and the solid lattice.

At a surface, there are two main channels for energy deposition, namely phonons and electron-hole pairs excitation. Phonons are commonly understood to be the main channel for dissipation [5]. The present authors' original conjecture is that during the interaction between molecule and phonons, 
there might be coupling between molecule vibration (characteristic interaction time) and surface vibration modes. Growing efficiency of this interaction (frequency matching) would then raise the transfer of energy to the surface. Therefore, one should know the phonon frequency distribution and compare it to the characteristic time of recombination of a molecule at the surface. This work has revealed an unexpected degree of physical and mathematical complexity.

Once issues 1 to 3 have been analyzed and recognized among the main contributors to the possible increase of $\beta$ with respect to the minimum defined by Eq. (2), the next step should be that of improving the modeling developed with the introduction of the following quantities: kinetic energy of impacting atom, interaction time, and the characteristic frequencies of the solid composing the outer titanium plasma-sprayed (TPS) surface.

The working assumption being investigated at this time is based on the observation that the longer the recombination process on the surface, the higher the probability of interaction with the surface. In particular, the longer the time the kinetic steps on the surface take, the higher the chance of depositing the $\mathrm{O}$ and $\mathrm{N}$ recombination heat in the form of phonons, rather than in the form of vibrational or electronic excitation of desorbing molecules.

It is this interaction that should lead to coupling with surface vibration modes and thus transfer energy from just-recombined species to the surface lattice.

The interaction time, in turn, seems to be dependent on the atom kinetic energy; in fact Erofeev [6] suggests to evaluate the interaction time as a ratio between a linear parameter related to the interaction potential and the atom impact velocity. Therefore, at this point, an adequate model of the interaction potential is needed.

\subsection{Potential Function for O Atoms Impacting Silica Surfaces}

In order to proceed, it is necessary to define the interaction model between the surface and the impacting atoms, that is the potential function.

For the $\mathrm{O} /$ silica system, due to the lack of spectroscopic data, a realistic interaction potential remains unknown and only simplified potential models are available.

The model used in this work is the two-body Morse-Stretch (MS) potential that is expressed as function of the distance $r$ between $A$ and $B$ atoms by

$$
V(r)=D_{e}\left[\left(1-\exp \left(\frac{\gamma}{2} \frac{R_{e}-r}{R_{e}}\right)^{2}-1\right)\right]
$$

where $D_{e}$ is the bond energy between $A$ and $B ; R_{e}$ is the equilibrium distance; and $\gamma$ is the parameter. 
Table 1 Morse parameters for $\mathrm{SiO}_{2}[8]$ and $\mathrm{O}-\mathrm{O}$ interaction [9]

\begin{tabular}{clcr}
\hline Interaction & $R_{e}, \AA$ & $D_{e}, \mathrm{~kJ} / \mathrm{mol}$ & $\gamma$ \\
\hline $\mathrm{Si}-\mathrm{O}$ & 1.6022 & 192.5894 & 8.8022 \\
$\mathrm{O}-\mathrm{O}$ & 1.526 & 69.928 & 11.4755 \\
\hline
\end{tabular}

For the definition of the potential parameters, the present authors followed the assumption in [7]: that is, the interaction of a Si lattice atom with a gas $\mathrm{O}$ atom is assumed equal to the $\mathrm{Si}-\mathrm{O}$ interaction in the lattice; that between the $\mathrm{O}$ gas atom and the $\mathrm{O}$ lattice atom is assumed to be the same as the $\mathrm{O}-\mathrm{O}$ interaction in gas phase. The Morse potential function parameters for $\mathrm{SiO}_{2}$, in the $\beta$-cristobalite form, are reported in Table 1 for the $\mathrm{Si}-\mathrm{O}$ interaction [8], together with those for $\mathrm{O}-\mathrm{O}[9]$.

Once the potential function is modeled, it is possible, in principle, to extract the following parameters needed by the kinetic catalysis model:

- the minimum, or minima, of the function, (the 'well' depth) corresponding to the bond energy and then to the adsorption energy;

- the minimum location(s) assumed the adsorption sites (the bottom of potential wells, where atoms can be trapped), providing also the intersite distance; and

- the difference between the minimum and the maximum value of the potential along the minimum energy path between two sites is assumed the migration energy.

Thus, in order to define the function in Eq. (3), a model of the atoms arrangement inside the lattice must be specified. When an atom 'hits a surface,' it is not subjected to the interaction of a single lattice atom, but of all lattice atoms within a certain distance. Therefore, the calculation domain for the potential function has been extended to a whole portion of the three-dimensional (3D) space surrounding the lattice and it is not limited to the first layer of $\mathrm{Si}$ atoms (the 'surface').

This approach allows evaluating possible sites everywhere inside the lattice not necessarily corresponding to intuitive or predefined locations. Here the lattice portion considered in the calculation is constituted by five atoms planes in the (111) direction (in the face-centered cubic (fcc) lattice, the close packed layers constitute, in the Miller notation, the $\{111\}$ group [10], see Fig. 2) and by three elementary tetrahedrals in the plane ( $x$ direction), as shown in Fig. 3. These planes constitute tetrahedral units. This ensemble of sites is periodic in 


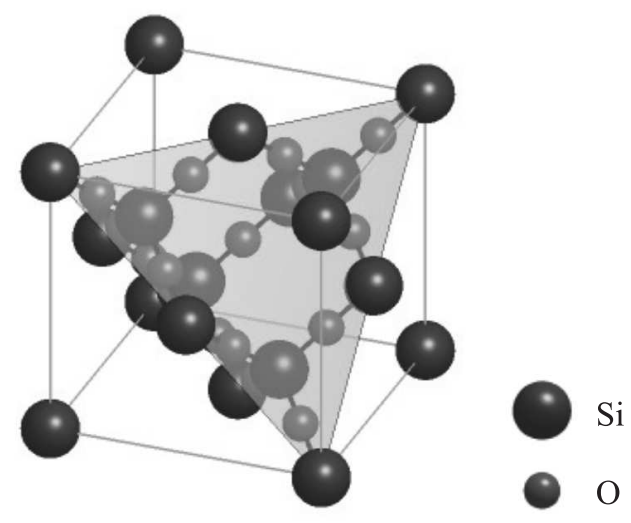

Figure 2 Unit cell and in grey the plane (111)

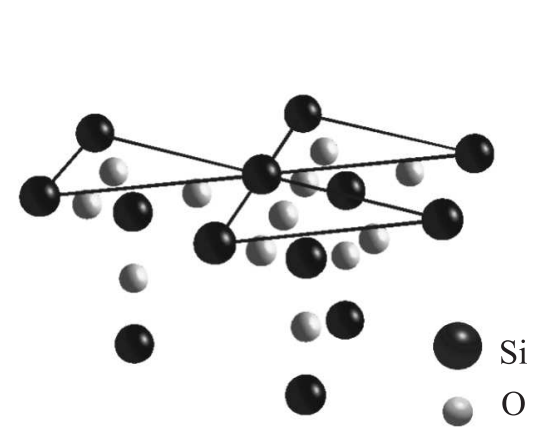

(a)

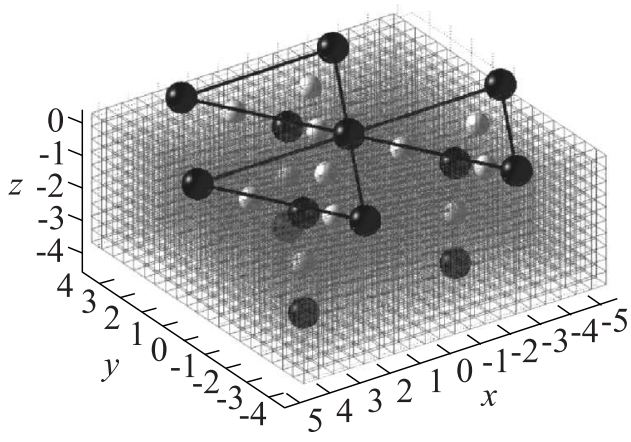

(b)

Figure 3 Five planes along the (111) direction (3D view) ( $a$ ) and grid for the potential computation $(b)$

the lattice, and is extended to calculate the potential as described in the following. Starting from Eq. (3), the potential $V(\vec{x})$ describing the interaction of the impinging atom with the portion of lattice considered, has been formulated by applying the superimposition principle, as linear summation of the contributions of each lattice atom:

$$
V(\vec{x})=\sum_{i=1}^{n} V_{i}\left(r_{i}\right)=\sum_{i=1}^{n} D_{e_{i}}\left[\left(1-\exp \left(\frac{\gamma}{2} \frac{R_{e_{i}}-r_{i}}{R_{e_{i}}}\right)^{2}-1\right)\right]
$$


where $n$ is the number of atoms in the portion of lattice and $r_{i}=\left|\vec{x}-\vec{x}_{i}\right|$ is the distance between the position $\vec{x}$ of the impinging atom and the position $\vec{x}_{i}$ of the $i$ th atom of the lattice.

The procedure for the potential computation has been implemented in Matlab language, the potential being defined by Eq. (4) with the coefficients of Table 1 and the computational grid built on the lattice structure of Fig. 3. By way of example, a grid constituted by $30 \times 30 \times 30$ nodes is shown in Fig. $3 b$; the number of nodes has been reduced from the one used only for the sake of clarity.

This procedure includes the effect on the potential of a whole portion of lattice and not only on the layer of atoms seemingly 'in contact' with the gas. The portion of lattice considered for the potential calculation is large enough to provide significant results and, in the $z$ direction, the minimum value does not change in a significant way when removing the last plane of atoms (third plane of $\mathrm{Si}$ atoms).

A different situation is verified in the $x y$ plane. In fact, the silicon atoms which form the vertices of the hexagon constituted by the top $\mathrm{Si}$ atoms in the

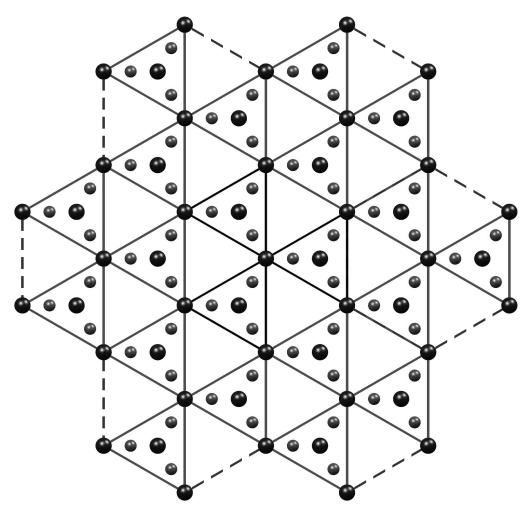

Figure 4 Extended portion of lattice (157 lattice atoms) $x y$ plane (grey in Fig. $3 b$ ), due to the lack of the adjacent lattice structure, have free bonds, thus altering the computation of the potential. To avoid this problem, the potential has been computed on the extended portion of lattice shown in Fig. 4, but choosing only the values obtained on the portion previously defined (see Fig. 3). The total number of lattice atoms considered is 157 . All the results presented in the following have been obtained with this procedure.

The absolute minimum of the function, which should be representative of the adsorption energy, is $388.64 \mathrm{~kJ} / \mathrm{mol}$. This value does not define a point in the lattice space, but it is possible to detect regions of absolute minima, which are inside the lattice, as clarified by the potential isosurfaces at $-380 \mathrm{~kJ} / \mathrm{mol}$ shown in Fig. 5.

Thus, by looking at the potential isosurfaces, a first interesting result is that the absolute minimum of the potential function, and thus possible adsorption sites, is inside the lattice, that is, at a certain distance from the first layer of atoms. This means that if the gas atom has enough energy, it can penetrate the lattice and be chemisorbed.

Once the absolute minimum of the function and its locations have been defined, local minima of the function have been evaluated in correspondence of 


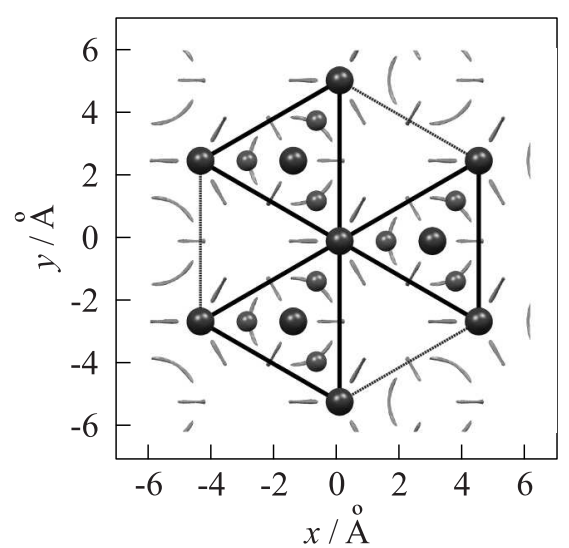

(a)

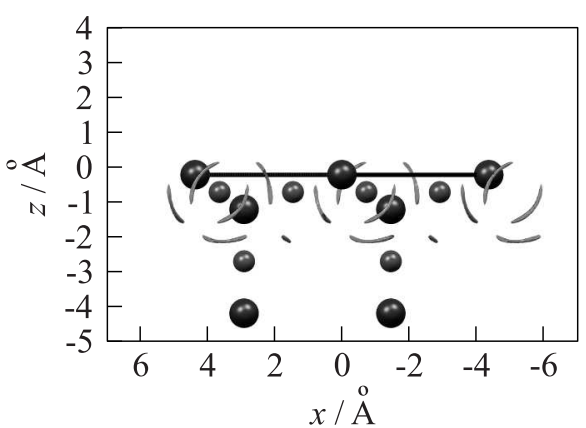

(b)

Figure 5 Potential isosurfaces at $-380 \mathrm{~kJ} / \mathrm{mol}$ : top $(a)$ and side $(b)$ views

each grid station at fixed $(x, y)$ values and with $z$ going from $2 \AA$ (above the surface), to the inner of the lattice at $-5 \AA$. The result is shown in Fig. 6. The positions of the minima inside the lattice, for each $(x, y)$ position, are visualized in Fig. 7.

$V / \mathrm{kJ} / \mathrm{mol}$

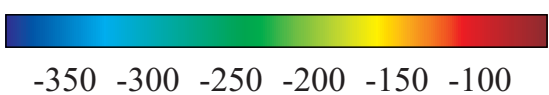

$\begin{array}{llllll}-350 & -300 & -250 & -200 & -150 & -100\end{array}$
$V / \mathrm{kJ} / \mathrm{mol}$
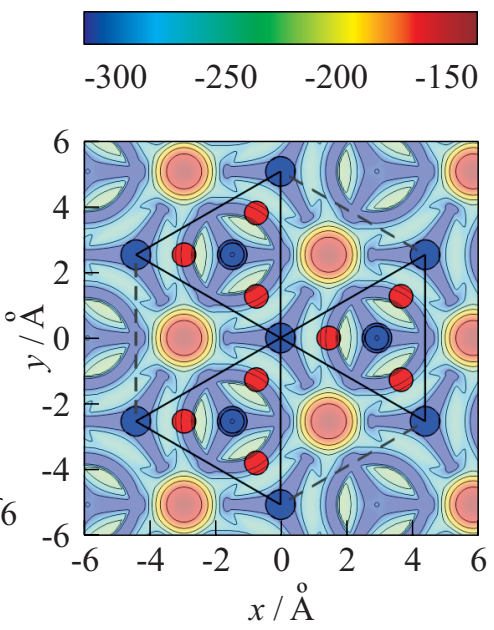

(b)

Figure 6 Value of the absolute minimum along $z$ for each $(x, y)$ position: 3D $(a)$ and top $(b)$ views. 


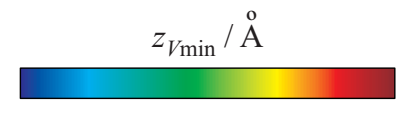

$\begin{array}{llllllll}1.0 & 0.5 & 0.0 & 0.5 & 1.0 & 1.5 & 2.0\end{array}$

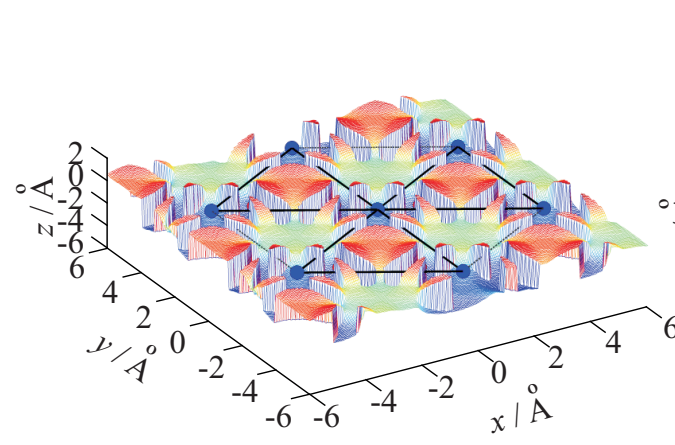

(a)

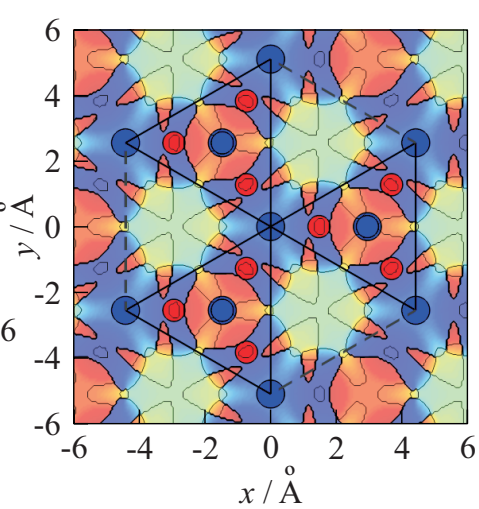

(b)

Figure 7 Positions of the minima inside the lattice: 3D $(a)$ and top $(b)$ views.

With the aim of defining the possible sites of adsorption, and then the path of minimum energy of an atom migrating on the surface, besides the absolute minimum of the potential function, some regions of potential values close to the minimum and some significant locations have been identified. They are indicated in Fig. 8 with capital letters. The same letters indicate equivalent energy positions inside the lattice.

The letter $C$ represents the absolute minimum positions and therefore (plausibly) the preferential adsorption sites. In correspondence of these points, the monodimensional distributions along the $z$ coordinate (see Fig. 8) allow to visualize the potential curve in a simpler way and to identify the potential well positions inside the lattice. Same potential profiles correspond to equivalent positions.

From Fig. 8, the following conclusions can be drawn:

- as already said, the absolute minimum energy positions for a particle impacting the surface are constituted by the potential wells positioned inside the lattice, but very close to the surface and between two adjacent Si atoms $(C$ positions in Fig. 8). The calculated minimum value can be assumed $Q_{a}=388.64 \mathrm{~kJ} / \mathrm{mol}$;

- at each site corresponds a different $z$ location in the lattice, i. e., the sites are not all at the same distance from the apparent surface in contact with the gas flow; 


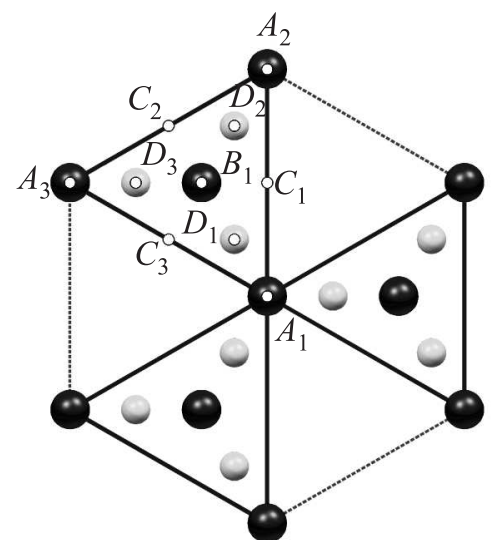

(a)

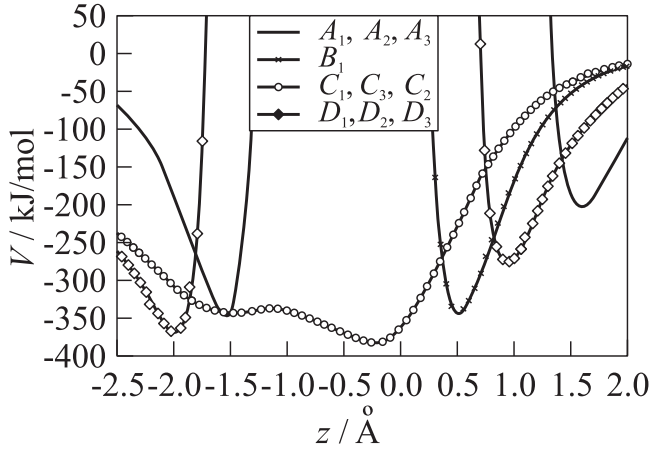

(b)

Figure 8 Positions of the minima inside the lattice (1) and monodimensional potential profiles $(b)$

- at positions indicated with $A$, the possible sites for $\mathrm{O}$ adsorption are located at a distance from the $\mathrm{Si}$ atom of the first plane $(z=0)$ similar to the $\mathrm{Si}-\mathrm{O}$ bond length. These sites are located either on the top of the first layer or inside the lattice, but of the two conditions, the latter should be the most probable, the well being at lower potential; and

- these points may be connected by a path that can be assumed as that of minimum energy, and that therefore gives information about migration energy and number of sites.

These conclusions suggest some changes to the original parameters characterizing the original silica phenomenological model [2]. In particular, once the 3D potential surface has been constructed and the possible adsorption sites visualized, the following considerations on adsorption energy, density of sites, distance between adjacent sites, migration energy and activation energy for LH recombination can be made.

For adsorption energy, one can naturally assume the potential value in correspondence of the absolute minimum of the function: $Q_{a}=388.64 \mathrm{~kJ} / \mathrm{mol}$. The density of sites needs instead further modeling and considerations:

- assume as adsorption sites those indicated in Fig. 8;

- with the exception of point $B_{1}$, there are always triplets of sites $\left(A_{1}-A_{2}-\right.$ $\left.A_{3}, C_{1}-C_{2}-C_{3}, D_{1}-D_{2}-D_{3}\right)$ positioned at the same $z$ coordinate inside the lattice, suggesting a density of three sites on a surface equal to that of the triangle $A_{1} A_{2} A_{3}$ is a plausible assumption; and 
- from the adsorption sites arrangement in the hexagon of Fig. 8, it is clear that three triangles out of six must be recognized to form each hexagon; then the density defined in the previous point should be divided by 2 .

To obtain the triangle area:

- hexagon side:

$$
l=a \frac{\sqrt{2}}{2}=7.15 \frac{\sqrt{2}}{2}[\AA]=5.076[\AA] ;
$$

- triangle height:

$$
h=l \frac{\sqrt{3}}{2}=a \frac{\sqrt{6}}{4}=4.378[\AA]
$$

- triangle area:

$$
A=\frac{1}{2} l h=a^{2} \frac{\sqrt{3}}{8}=11.068\left[\AA^{2}\right]
$$

and, thus, for the density of sites:

$$
\rho=\frac{\# \text { sites }}{\text { Area }}=\frac{3}{2 A}=0.1355\left[\AA^{-2}\right]=1.355 \cdot 10^{19}\left[\mathrm{~m}^{-2}\right] .
$$

To evaluate the distance $\Delta$ between adjacent sites, calculate distances between equivalent sites in Fig. 8:

(a) $\Delta\left(\mathrm{A}_{i} \mathrm{~A}_{j}\right)=5.076[\AA]$;

(b) $\Delta\left(\mathrm{C}_{i} \mathrm{C}_{j}\right)=a \sqrt{2} / 2 \sin (\pi / 6)=2.528[\AA]$; and

(c) $\Delta\left(\mathrm{D}_{i} \mathrm{D}_{j}\right)=\Delta\left(\mathrm{C}_{i} \mathrm{C}_{j}\right)=2.528[\AA]$.

The distance (a) is that assumed in the silica original model [2], but in this work, the value $2.528 \AA$ seems the most probable. In this evaluation, the fact that adatoms might migrate also in the $z$ direction of the lattice has been neglected; if not, the distance would be even shorter. For the computation of the migration energy, refer to the monodimensional potential profiles in Fig. 8. It is impossible to define just one migration path trough the sites just identified; this statement is made stronger by the observation that the sites are located at different distance from the surface, thus increasing the possibility of migration within the lattice.

For the given purpose, the final aim is to compute the LH recombination activation energy, defined as:

$$
Q_{\mathrm{LH}}=\max \left(2 Q_{a}-D, E_{m}\right)
$$


where $Q_{a}$ is the adsorption energy; $D$ is the dissociation energy; and $E_{m}$ is the migration energy [11]. The constraint above leads to compute the migration energy as difference between the potential energies of the sites corresponding to the greatest energy 'jump.' These sites are $A_{i}$ and $C_{i}$ and, thus, for the migration energy, one has:

$$
E_{m}=\operatorname{abs}\left[V\left(C_{i}\right)-V\left(A_{i, z=1.5}\right)\right] \operatorname{abs}(-388.64+202)=186.64[\mathrm{~kJ} / \mathrm{mol}]
$$

and since $D_{\mathrm{O}_{2}}=498 \mathrm{~kJ} / \mathrm{mol}$, the $Q_{\mathrm{lh}}$ is

$$
Q_{\mathrm{lh}}=2 Q_{a}-D=279.28[\mathrm{~kJ} / \mathrm{mol}] \text {. }
$$

Finally, the value of $Q_{\mathrm{LH}}$ is about half of the value in the original model $\left(Q_{\mathrm{LH}}=501.6 \mathrm{~kJ} / \mathrm{mol}\right.$ in $\left.[2]\right)$ :

$$
Q_{\mathrm{LH}}=\max \left(Q_{\mathrm{lh}}, E_{m}\right)=279.28[\mathrm{~kJ} / \mathrm{mol}] .
$$

\section{NEW CHEMICAL ENERGY ACCOMODATION MODEL}

The definition 'minimum value' used so far means that Eq. (2) accounts only for the fraction of heat released to the surface by the adsorption processes and that the molecules resulting from wall recombination escape with all their initial energy $E=D-Q_{a}$ (adsorbed atoms use the quantity $Q_{a}$ to escape the potential well).

Thus, to complete the model, it is necessary to evaluate the fraction $\alpha$ of energy $E$ which is released at the wall and add the quantity $E_{\text {rec-wall }}=\alpha(D$ $-Q_{a}$ ) to the numerator of Eq. (4). To do this, start from the discussion in subsection 2.2 and, in particular, from item 2, the lifetime of the molecule before desorption, and item 3 , the coupling between the vibrational frequency of recombining molecule and that of the solid lattice.

In [1], the assumption that the molecules produced by recombination escape with all their initial energy is equivalent to assume that just-formed molecules transfer their energy on a time scale long compared with that of the other processes. But it is known, both from experiments [4] and monodimensional simulations $[3,12]$, that part of this energy is released to the wall and, therefore, that the interaction time is comparable with the time of other processes; in particular, in the present authors' original idea (see item 3), the efficiency of this interaction depends on the coupling between the molecules interaction time before desorption and the phonon frequency distribution (characteristic period of surface vibration). So, the first thing to do is to evaluate these two quantities for the case of $\mathrm{O}$ impacting on silica. 
An expression for the interaction time of a gas atom with a surface is provided by Erofeev in [6] as $\tau=c / V_{0}$ where $c$ is one of the parameters of the interaction potential, expressed by the Morse function in the form:

$$
U(r)=D\left[e^{-2(r-b) c^{-1}}-2 e^{-(r-b) c^{-1}}\right] ;
$$

therefore, the $c$ parameter represents a characteristic distance of the interaction, and $V_{0}$ is the atom initial velocity.

For what concerns the definition of $c$, one can take advantage of the 3D potential function defined in subsection 2.3; this function is, however, a sum of terms of the kind in Eq. (5). To extract a single constant, one may reproduce the values of interest of the $3 \mathrm{D}$ function by means of a one-dimensional (1D) potential in correspondence of a position of particular interest in the lattice. To this purpose, take the position of absolute minima in the lattice, labelled with $C_{i}$ in Fig. 8: isolate the potential function profile at these positions and reproduce the same potential well with the 1D function (5), as shown in Fig. 9.

The parameters found for the one-dimensionalized Morse function in this way are: $b=-0.1564$ and $c=0.58309 \AA$.

As for the $\mathrm{O}$ atom velocity, one can take the average value of the velocity provided by kinetic theory [13]:

$$
V_{0}=\sqrt{\frac{k T_{w}}{2 \pi m_{0}}} .
$$

For example, for $T_{w}=1500 \mathrm{~K}$, the velocity is $1106.5 \mathrm{~m} / \mathrm{s}$, the interaction time is $\tau=5.27 \cdot 10^{-14} \mathrm{~s}$ and the interaction frequency $\nu=1.89 \cdot 10^{13} \mathrm{~Hz}=632.99 \mathrm{~cm}^{-1}$.

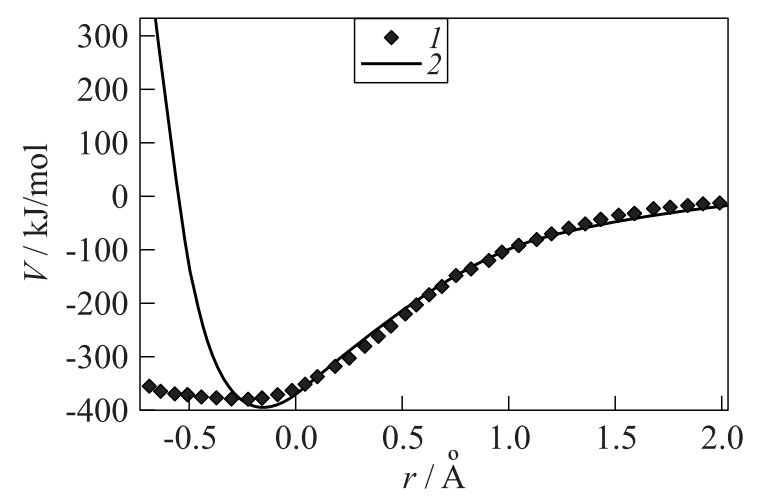

Figure 9 Portion of Morse potential 3D function (1) and its monodimensional approximation (2) 


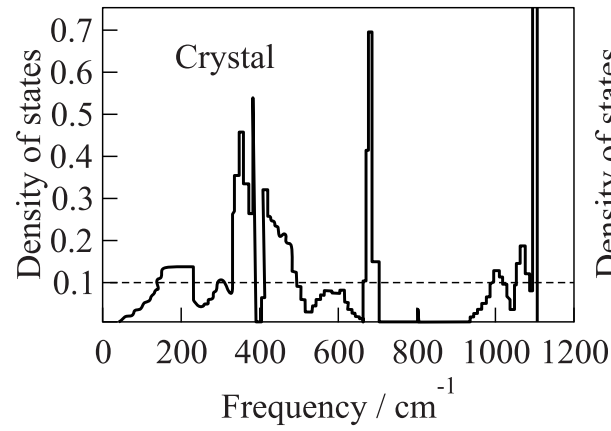

(a)

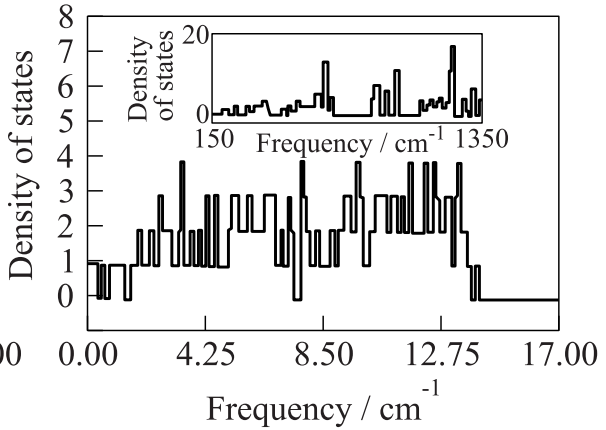

(b)

Figure 10 Density of states for $\mathrm{SiO}_{2} \beta$-cristobalite: from [14] (a) and from [7] (b)

This time must be compared with the vibrational spectra of surface phonons. In the open literature data on silica, vibrational spectra already exist; the spectra reported in Fig. 10 are by Sen and Thorpe [14] and by Cacciatore et al. [3].

In both studies, the frequency $\nu \sim 630 \mathrm{~cm}^{-1}$ (interaction frequency) corresponds to a normalized density of states $\approx 0.1$ (the density of states is defined as the number of phonons frequency in a certain interval of frequencies, see Fig. 8).

This value is a measure of the coupling between the vibrational frequency of recombining molecule and the solid lattice; in our model it is assumed as a measure of the fraction of the total energy $E=D-Q_{a}$ that is released at the wall by just recombined molecules, i.e., in the particular case analyzed, $E_{\text {rec_wall }}=0.1\left(D-Q_{a}\right)$.

The term $Q_{a}$ in the previous expression needs to be specified for the two recombination processes, ER and $\mathrm{LH}$, accounting for the probability of each process, the number of sites involved, and remembering that $\mathrm{LH}$ recombination frees two sites at one time. Therefore, one has: for $\mathrm{ER}, Q_{a} \theta_{\mathrm{O}} \gamma^{*}$; for $\mathrm{LH},(1 / 2) Q_{a} \theta_{\mathrm{O}}^{2} \sigma$, and for $E_{\text {rec_wall, }}$

$$
E_{\text {rec_wall }}=\alpha\left(\gamma \frac{D}{2}-Q_{a} \theta_{\mathrm{O}} \gamma^{*}-\frac{1}{2} Q_{a} \theta_{\mathrm{O}}^{2} \sigma\right) .
$$

Finally, let find the chemical energy accommodation coefficient:

$$
\begin{aligned}
\beta=( & Q_{a} s_{a}\left(1-\theta-\theta_{\mathrm{OH}}\right)-Q_{R} \gamma^{*} \theta-\frac{1}{2} Q_{\mathrm{LH}} \sigma \theta^{2}-Q_{a} \delta \theta \\
& \left.+\alpha\left(\gamma \frac{D}{2}-Q_{a} \theta_{\mathrm{O}} \gamma^{*}-\frac{1}{2} Q_{a} \theta_{\mathrm{O}}^{2} \sigma\right)\right) /\left(\left[s_{1}(1-\theta)-\delta \theta+\gamma^{*} \theta\right] \frac{D}{2}\right) .
\end{aligned}
$$


With this expression, it is now possible to compute the effective recombination coefficient $\gamma^{\prime}=\gamma \beta$.

\section{Validation Test}

The new catalytic recombination model for silica surfaces, with the parameters defined by means of the 3D potential function (see subsection 2.3) and the effective recombination defined in the previous section have been applied to significant cases and compared to experimental data on silica-based surfaces.

Note that the new model including 3D potential function and chemical energy accommodation modeling has been developed to treat only $O$ recombination, which is the most significant catalytic reaction in Low Earth Orbit (LEO) reentry.

As no flight measurements on silica are available yet, and therefore validation has been carried out using data collected during a test campaign, in the CIRA SCIROCCO PWT, on a full-scale spacecraft component exposed to the hypersonic airstream, with an additional complication due to the arc heater air vitiation. Thus, the presence of nitrogen atoms in the PWT is nonnegligible and catalytic formation of $\mathrm{N}_{2}$ and $\mathrm{NO}$ should be considered for more accurate results.

To address this issue, the following assumptions for the catalytic recombination of $\mathrm{N}$ atoms have been made:

- for what concerns the model parameters deduced by the potential function, the values of the original silica model [2] have been maintained; and

- the CEA of recombining $\mathrm{N}$ atoms has been modelled assuming both the same $\alpha$ coefficient of $\mathrm{O}$ and full accommodation $(\beta=1)$.

Finally, the silica-based material tested in PWT is proprietary, and different from the ideal surface of the present work.

The test has been carried on in the SCIROCCO PWT [15-17], under the ESA FLPP project, using a large TPS sample article designed and manufactured by SPS (Snecma Propulsion Solide), to validate the behavior of the assembly during reentry.

The test article is shown in Fig. 11; the parts to be tested are constituted by three panels made of Ceramic Matrix Composite (CMC), a silica-based proprietary material. The panels are in white in Fig. 11.

The test was designed to have a cold-wall $\left(T_{w}=300 \mathrm{~K}\right)$ heat flux equal to $320 \pm 10 \% \mathrm{~kW} / \mathrm{m}^{2}$, obtained imposing fully catalytic (FC) wall, in the region where the panels become flat, and a constant wall pressure 25 mbar on the two flat panels surface. 

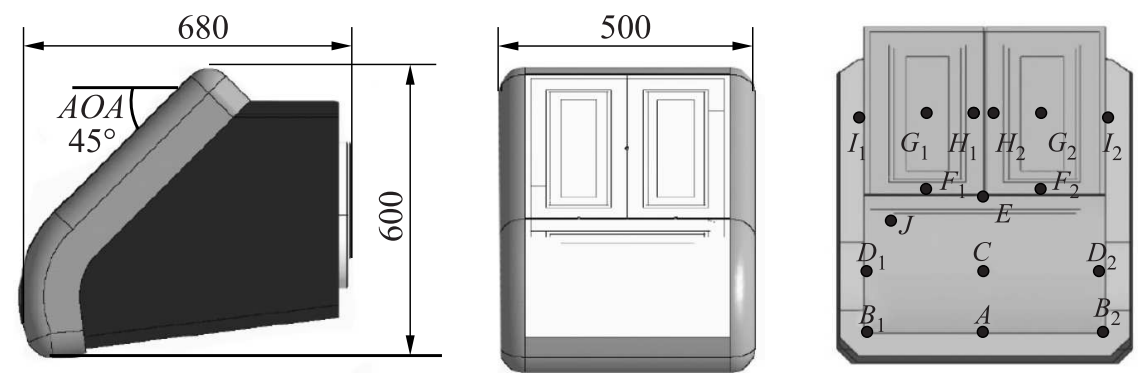

Figure 11 Test Article geometry and thermocouples location. Dimensions are in millimeters

The FLPP-SPS plasma test was performed in three steps, each characterized by increasing total enthalpy in the test chamber, i. e., the wall heat flux. The second test step, defined 'nominal,' has been rebuilt after the test by using as input data measured on the PWT SCIROCCO calibration probe stagnation point before the insertion of the model: these data are $P_{S}=34.20 \pm 1.1 \operatorname{mbar}_{a}$ and $Q_{S}=2120 \pm 90 \mathrm{~kW} / \mathrm{m}^{2}$.

During the test, eleven B-type thermocouples measured the back wall temperatures of the CMC panels. Those that have correctly worked $\left(F_{2}, G_{2}, H_{2}\right.$, and $H_{1}$, see Fig. 11) have been selected to compare their temperatures with those predicted by the Computational Fluid Dynamics (CFD) CIRA code.

The test chamber conditions rebuilt by CFD are: $P_{0}=4.9$ bar and $H_{0}$ $=17.4 \mathrm{MJ} / \mathrm{kg}$. Computational grid and heat flux contour map are shown in Fig. 12. The simulations have been performed with the CIRA CFD code H3NS [18].

The long duration of the test (about $15 \mathrm{~min}$ ), the small thickness of the CMC panels and the presence of an insulation layer behind them allow to assume radiative equilibrium at the wall $(\varepsilon=0.8)$, thus neglecting the thermal conduction inside the material. This assumption is confirmed by the thermocouples measurement history.

During the CFD rebuilding, the effect of wall catalysis was addressed in a very simplified way, by comparing the experimental temperature with both noncatalytic (NC) and FC predictions. The difference observed between actual temperature and that computed underlined the importance of proper modeling wall catalysis. The same case has been rebuilt in the present (and most recent) work by implementing the new catalysis model just described.

The result in terms of $\mathrm{O}$ CEA coefficient is shown in Fig. 13; the values predicted by the model are plotted along the $x$ section marked by a white line in the box reported in figure. Comparison between the $\mathrm{O}$ recombination coef- 

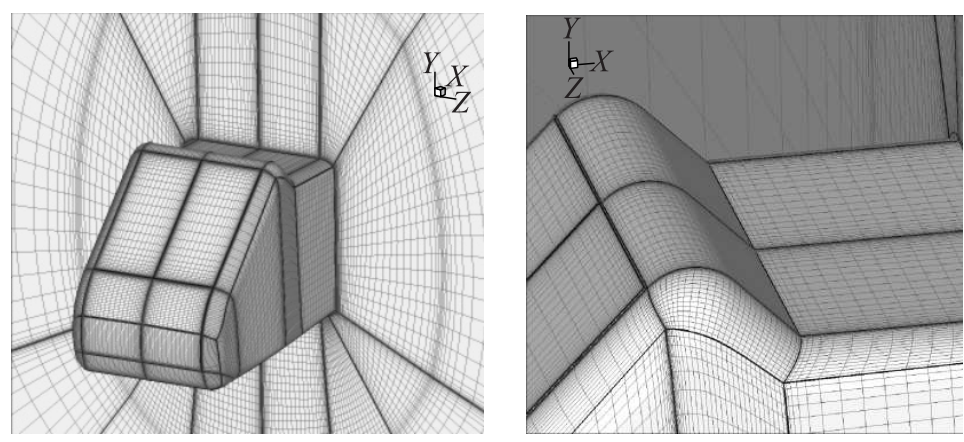

(a)

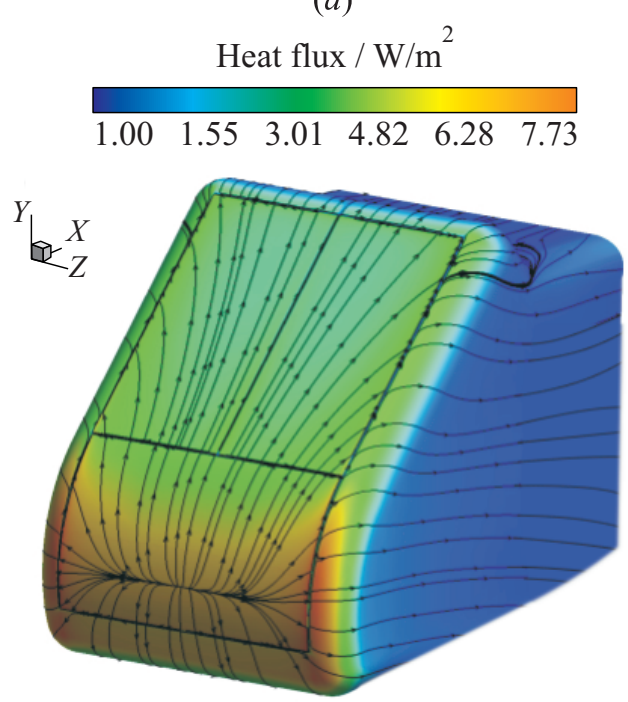

(b)

Figure 12 Computational grid on the test article configuration $(a)$ and heat flux contour map with skin friction lines $(b)$.

ficient with and without the effect of CEA is shown in Fig. 13b; comparison of temperature is shown by dashed lines in Fig. $13 b$.

Temperatures have been extracted from thermocouples measurements at positions $G_{2}, H_{2}$, and $H_{1}$ (see Fig. 11) located in the middle of the flap panels (Slice \# 1), and $F_{2}$ located at the origin of the panels (Slice \#2).

The results are presented in Fig. 14: those using the new catalysis model including the effect of CEA are marked with 3 ; curve 4 assumes full accommo- 


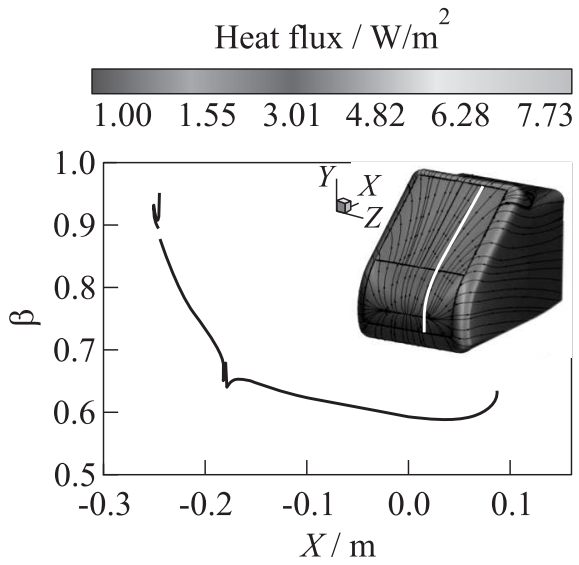

(a)

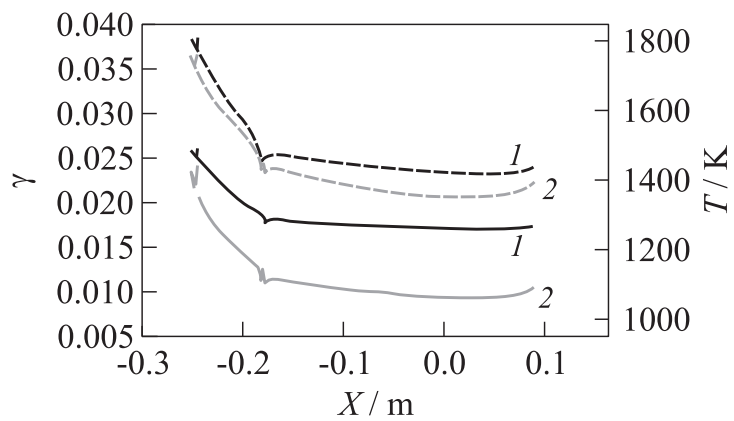

(b)

Figure 13 Oxygen CEA coefficient $(a)$ and oxygen recombination coefficient $\gamma$ (solid curves) and temperature (dashed curves) (b); distributions along the white $x$ section reported in the box. Comparison between partial and full accommodation: 1 - silica $\beta=1 ;$ and $2-$ silica $\beta$ model

dation, curves 1 and 2 are, respectively, obtained with the simplified assumption of FC and NC wall. Symbols indicate experimental data. Note that no error bars are reported for the temperature data, the measurement error being negligible for the type of thermocouples adopted $( \pm 1.1 \mathrm{~K}[15])$.

The agreement between temperatures measured and predicted with the new catalysis model (curve 3) is surprisingly good, especially for the first slice (Fig. 14a), and confirms that the new model simulating the catalytic behavior of silica-based CMC materials is useable.

In the second slice (Fig. 14b), the experimental temperature is better reproduced by full energy accommodation; this could be explained as an effect of 


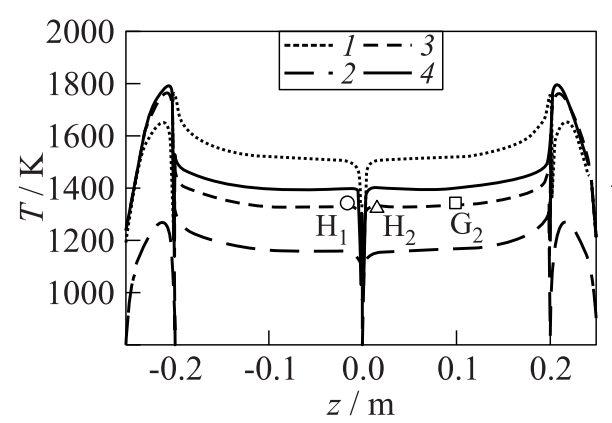

(a)

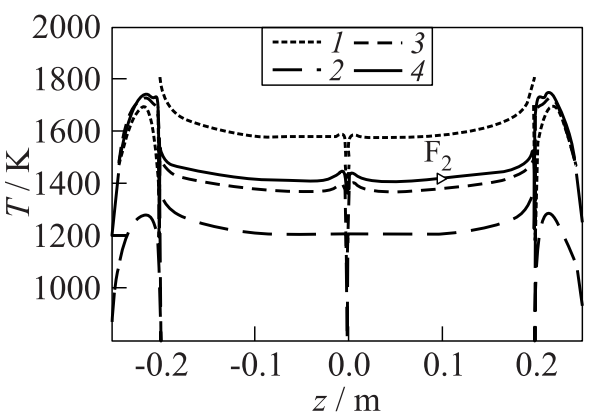

(b)

Figure 14 Measured and predicted temperature; finite (3) and complete accommodation (4) for Slice \# $1(a)$ and Slice \# $2(b)$; CFD: 1 - FC wall; 2 - NC wall; $3-$ FRC CEA model; and $4-$ FRC original silica model

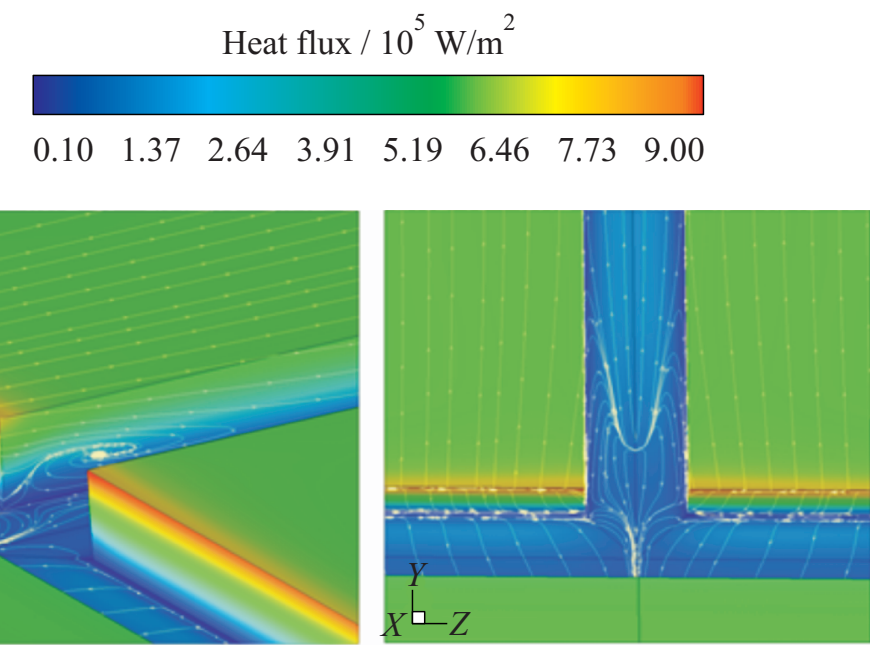

Figure 15 Heat flux contour map and flow structure in the $T$ gap.

temperature conduction from the gap edge (where a heat flux peak is predicted, see Fig. 15), not accounted for in the numerical rebuilding.

In comparison, both $\mathrm{FC}$ and $\mathrm{NC}$ temperature predictions are poor. 


\section{CONCLUDING REMARKS}

Modeling of partial chemical energy accommodation has been identified as a priority need in trying to improve existing kinetic catalysis model.

Two different approaches have been presented for the CEA coefficient modeling. The first has produced a simplified expression for the minimum estimate of the chemical accommodation coefficient $\beta$, corresponding to the case where recombined molecules desorb without depositing extra energy on the surface. This is the case where only adsorption is responsible for energy deposition. This simplified $\beta$ has been included in the kinetic model and validated with experimental wind tunnel data, providing interesting results.

The second approach is based on a novel idea, that is, the linking the additional release of energy (with respect to the minimum value found), to the following quantities: the kinetic energy of the atom impacting the surface; the lifetime of the molecule before desorption; and, especially, the coupling between the vibrational frequency of recombining molecule and that of the solid lattice. The latter assumes most naturally that the energy would therefore be transferred to the surface by means of phonons vibration. In this second formulation, a fundamental role will be played by the new potential model developed and by information coming from monodimensional simulations.

Moreover, a new 3D potential function has been defined. The evaluation of a suitable potential function, even obtained by a simplified approach, is of fundamental importance: it allows to find the surface sites where gas atoms will probably form a chemical bond with the surface. This is important, as most of the kinetic model parameters depend on location and energy of well sites. From this study, more realistic parameters for the kinetic model have been extracted.

The new model was implemented in a Navier-Stokes CFD code, and allows the prediction of the effective recombination coefficient $\left(\gamma^{\prime}=\gamma \beta\right)$, and, thus, the prediction of more realistic heat loads, enabling the preliminary design of a better (lighter) TPS.

Due to the lack of flight measurements, the validation of the model has been carried out using data collected during a test campaign in the CIRA PWT SCIROCCO. The agreement between the temperatures measured and those predicted by CFD with the new catalysis model is good, thus confirming the model can simulate the catalytic behavior of silica-based materials.

Reproducing experimental measurement with CFD is the key to "extrapolating to flight" any PWT results. Since the flight environment is different from that of a PWT (different amount of chemical energy available for recombination, different Damköhler numbers), reproducing the material temperature during tests also depends on accurate knowledge of the material catalytic behavior.

The widespread custom of assuming fully catalytic TPS can lead to wrong conclusions with respect to the material qualification, and also wrong aerody- 
namics predictions, such as the location of the center of pressure and the pitching moment.

Finally, the availability of a partially validated analytical model, applicable to a class of materials, may allow to reduce conservative margins in the design of TPS for space applications. Note that the TPS constitutes a significant fraction of the mass budget for any reentry vehicle: thus relaxing, the fully catalytic assumption may allow a significant increase in payload mass.

\section{REFERENCES}

1. Halpern, B., and D. E. Rosner. 1978. Chemical energy accommodation at catalyst surfaces. Chem. Soc. Faraday Trans. 1 74:1833-912.

2. Nasuti, E., M. Barbato, and C. Bruno. 1996. Material-dependent catalytic recombination modeling for hypersonic flows. J. Thermophys. Heat Transfer 10(1):131-36.

3. Cacciatore, M., M. Rutigliano, and G. D. Billing. 1999. Eley-Rideal and LangmuirHinshelwood recombination coefficients for oxygen on silica surfaces. J. Thermophys. Heat Transfer 13(2):195-203.

4. Balat-Pichelin, M., and L. Bedra. 2005. Combined approach for the experimental evaluation of the heterogeneous catalycity parameters under atmospheric re-entry conditions. 1st EUCASS Conference. Moscow, Russia.

5. Groß, A. 2006. Simulation of gas-surface dynamical interactions. RTO-AVT-VKI lecture ser.

6. Erofeev, A. 1966. Interaction of molecules with the surface of a solid. J. Appl. Mech. Techn. Phys. 3:42-49.

7. Cacciatore, M., and M. Rutigliano. 2007. Molecular dynamics simulations of surface processes: Oxygen recombination on silica surfaces at high temperatures. In: Experiment, modeling and simulation of gas-surface interactions for reactive flows in hypersonic flights. RTO-EN-AVT-142. Paper 5.

8. Demiralp, E., T. Cagin, and W. A. Goddard III. 1999. The MS-Q force field for ceramics; application to the quartz-stishovite phase transition and to silica glass. Phys. Rev. Lett. 82(7):1708-11.

9. Konowalow, D. D., and J. O. Hirschfelder. 1961. Morse potential parameters for $\mathrm{O}-\mathrm{O}, \mathrm{N}-\mathrm{N}, \mathrm{N}-\mathrm{O}$ interactions. Phys. Fluids 4(5):637-42.

10. Sands, D. E. 1993. Introduction to crystallography. In: Crystal system and geometry. Dover Publications.

11. Di Benedetto, S. 2011. Modelling of gas-surface interaction phenomena in hypersonic flight. Ph.D. Dissertation. Rome, Italy: University of Rome 'La Sapienza,' Facoltà di Ingegneria.

12. Kovalev, V. L., and M. Ju. Pogosbekian. 2008. Catalytic properties analysis of the heat shield materials by molecular dynamic methods. 6th European Symposium on Aerothermodynamics for Space Vehicles. Versailles, France.

13. Moelwyn-Hughes, E. A. 1964. Physical chemistry. 2nd ed. Pergamon Press. 
14. Sen, P.N., and M.F. Thorpe. 1977. Phonons in AX2 glasses: From molecular to band-like modes to band-like modes. Phys. Rev. B 15(8):4030-38.

15. Trifoni, E., F. Alfano, D. Mandanici, et al. 2007. PWT Test Report. CIRA-TR-070230 .

16. Rufolo, G. C., S. Di Benedetto, and M. Marini. 2008. Theoretical-numerical design of a plasma wind tunnel test for a large TPS demonstrator. 6th European Symposium on Aerothermodynamics for Space Vehicles. Versailles, France.

17. Di Benedetto, S., G. Rufolo, M. Marini, and E. Trifoni. 2008. Aerothermodynamic analysis of a Scirocco PWT on a large TPS demonstrator and comparison with experimental results. 6th European Symposium on Aerothermodynamics for Space Vehicles. Versailles, France.

18. Ranuzzi, G., and S. Borreca. 2006. H3NS: Code development and validation. CLAE Project. CIRA-CF-06-1017. 